\title{
Behavior of Plasma-doped Graphene upon High Temperature Vacuum Annealing
}

\author{
Byeong-Joo Lee, Sung-Il Jo, and Goo-Hwan Jeong* \\ Department of Advanced Materials Science and Engineering, Kangwon National University, Chuncheon, \\ Gangwon-do 24341, Republic of Korea
}

Received July 30, 2018; revised September 28, 2018; accepted September 29, 2018

\begin{abstract}
Herein, we present the behavior of plasma-doped graphene upon high-temperature vacuum annealing. An ammonia plasma-treated graphene sample underwent vacuum annealing for $1 \mathrm{~h}$ at temperatures ranging from 100 to $500{ }^{\circ} \mathrm{C}$. According to Raman analysis, the structural healing of the plasma-treated sample is more pronounced at elevated annealing temperatures. The crystallite size of the plasma-treated sample increases from 13.87 to $29.15 \mathrm{~nm}$ after vacuum annealing. In addition, the doping level by plasma treatment reaches $2.2 \times 10^{12} \mathrm{~cm}^{-2}$ and maintains a value of $1.6 \times 10^{12} \mathrm{~cm}^{-2}$, even after annealing at $500{ }^{\circ} \mathrm{C}$, indicating high doping stability. A relatively large decrease in the pyrrolic bonding components is observed by X-ray photoelectron spectroscopy as compared to other configurations, such as pyridinic and amino bindings, after the annealing. This study indicates that high-vacuum annealing at elevated temperatures provides a method for the structural reorganization of plasma-treated graphene without a subsequent decrease in doping level.
\end{abstract}

Keywords: Graphene, Plasma doping, Vacuum annealing, Structure healing, Doping stability

\section{Introduction}

Due to its zero-bandgap character, it is necessary to modify the electronic properties of graphene, such as charge doping level, charge carrier mobility, and bandgap width, to realize extensive electronic applications $[1,2]$. Some strategies have been reported to control the physical properties of graphene through structural modification and chemical doping [3-5]. For example, Silva et al. reported structural graphitization and edge loop formation of graphene nanoribbons by high-temperature annealing above $2800{ }^{\circ} \mathrm{C}$ [4]. A layer-by-layer thinning of multilayered graphene via plasma treatment and consecutive annealing has been reported [5]. However, due to the difficulty of executing precise control over the graphene nanostructure, many studies use thermal- and plasma-based methodologies for physical property modification. Jiao et al. reported the reduction and nitrogen doping of graphene oxide by thermal treatment at $300-1100{ }^{\circ} \mathrm{C}$, using a mixture of $\mathrm{H}_{2}$ and $\mathrm{NH}_{3}$ [6]. Li et al. also demonstrated nitrogen doping of graphene at $900{ }^{\circ} \mathrm{C}$, using $\mathrm{NH}_{3}$ [7]. Plasma doping has also been applied as a quick and low temperature process. Lin et al. demonstrated graphene doping using $\mathrm{NH}_{3}$ plasma at

*Corresponding author

E-mail: ghjeong@kangwon.ac.kr room temperature [8]. Xie et al. also reported the quick hydrogenation of graphene using $\mathrm{H}_{2}$ plasma [9]. However, the structural degradation caused by high energy species during plasma processing remains an inherent problem to overcome for the realization of an efficient, functional doping process and graphene-based device applications. It is very important to develop a method to preserve the structure of graphene during the doping process, or to restore the damaged carbon networks. This is because the performance of a graphene-based device is influenced by the formation of structural defects, when graphene is exposed to plasma or heat during device operation. To systematically investigate the behavior of structural healing and thermal stability of the plasma-doped graphene, plasma doping and subsequent vacuum annealing at elevated temperatures is performed.

\section{Materials and methods}

A highly crystalline graphene sample was obtained by mechanical exfoliation from kish graphite. The prepared graphene sample was exposed to ammonia plasma for nitrogen doping using parallel plate direct-current plasma system. The ammonia plasma was ignited at 0.3 Torr with an applied bias voltage of $450 \mathrm{~V}$, and the exposure time was fixed to $10 \mathrm{~s}$. The detail process of plasma doping is 
described elsewhere [10]. After the plasma treatment, we subsequently purged the chamber twice with ambient $\mathrm{Ar}$, below $10^{-6}$ Torr, using a turbomolecular pump. Then, the substrate was heated to the programmed annealing temperature (ranging between $100-500{ }^{\circ} \mathrm{C}$ ) for $1 \mathrm{~h}$. The chamber pressure was maintained below $10^{-6}$ Torr.

The plasma-doped and vacuum-annealed graphene was characterized using Raman scattering spectroscopy (Renishaw) and X-ray photoelectron spectroscopy (ThermoVG Sigma Probe, XPS) in the Central Laboratory of Kangwon National University [11]. For the Raman analysis, the spot size was $1-\mu \mathrm{m}$-diameter with an excitation wavelength of $532 \mathrm{~nm}$. The laser power was kept under $3.0 \mathrm{~mW}$ to avoid heatinginduced damage. The XPS spectra were acquired using a monochromated AlK $\alpha$ X-ray source with a spot diameter of $400 \mu \mathrm{m}$. The surface topography was measured using AFM (Park Systems XE-70) in tapping mode. The scan rate was maintained in the range of $0.5-1 \mathrm{~Hz}$.

\section{Results and discussion}

Raman scattering spectroscopy is a very useful tool to characterize not only the structural quality but also the chemical doping of graphene. In general, there are specific peaks in the Raman spectrum of graphene, namely the D-, G-, D'- and 2D-bands at 1350, 1580, 1620 and $2675 \mathrm{~cm}^{-1}$, respectively $[12,13]$. The $\mathrm{D}-$ and $\mathrm{D}^{\prime}$-bands are related to structural defects in graphene and the G-band presents inplane tangential vibration of $s p^{2}$ carbon atoms. Thus, the intensity ratio of the D- to G-bands $\left(\mathrm{I}_{\mathrm{D}} / \mathrm{I}_{\mathrm{G}}\right)$ and $\mathrm{D}^{\prime}$ - to Gbands $\left(\mathrm{I}_{\mathrm{D}} / \mathrm{I}_{\mathrm{G}}\right)$ can be used as an indicator of graphene crystallinity. The 2D-band, also called the G'-peak, is of second order to the D-band. This is a single Lorentzian peak in single layer graphene, and splits into four peaks in bilayer graphene, reflecting the evolution of the electron band structure. The number of graphene layers can be estimated by the intensity ratio of G- and $2 \mathrm{D}$-bands $\left(\mathrm{I}_{\mathrm{G}} /\right.$ $\mathrm{I}_{2 \mathrm{D}}$ ), and also by the peak position of the 2D-band $[12,13]$.

Figure 1(a) shows the Raman spectra for the asexfoliated and plasma-treated graphene. There are no Dand $\mathrm{D}^{\prime}$-bands, which means the prepared graphene is of high quality. In addition, the value of $\mathrm{I}_{\mathrm{G}} / \mathrm{I}_{2 \mathrm{D}}$ is 0.31 , indicating monolayer formation. After plasma treatment, the $\mathrm{D}$ - and $\mathrm{D}^{\prime}$-bands are observed, as well as an increase in the $\mathrm{I}_{\mathrm{G}} / \mathrm{I}_{2 \mathrm{D}}$ value to 0.64 . This means that the structural defects on graphene might be formed due to plasma ion bombardment.

Figures 1(b) and 1(c) display the AFM topographic images and height profiles for the as-fabricated and plasmatreated graphene, respectively. The surface roughness values $\left(\mathrm{R}_{\mathrm{a}}\right)$ are 0.43 and $0.45 \mathrm{~nm}$, meaning that the graphene structure is not significantly degraded after plasma treatment. The plasma conditions used in this study were optimized in a previous study to suppress high-energy ioninduced damage to yield efficient nitrogen-doped graphene
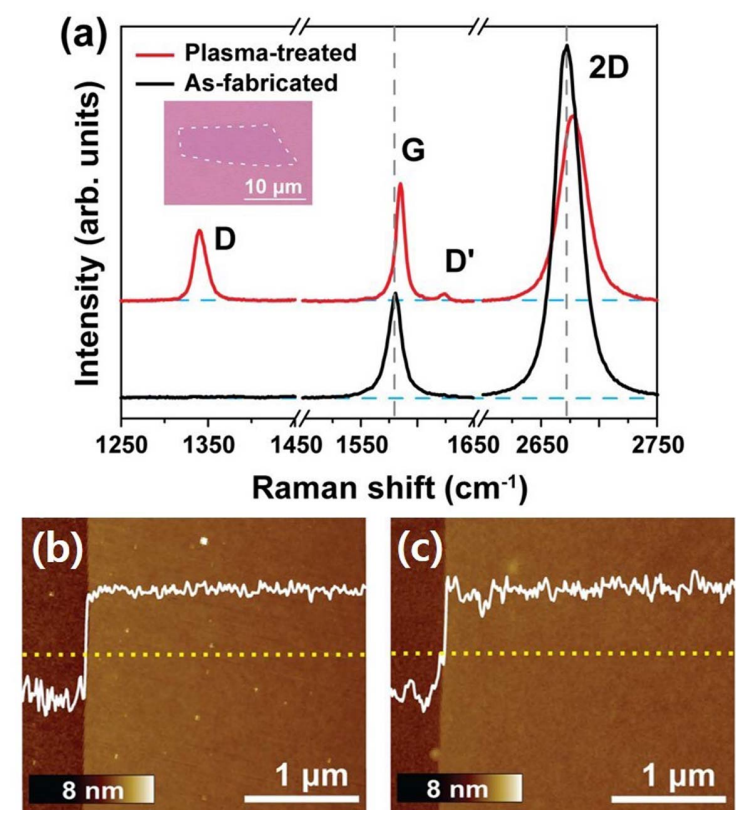

Figure 1. (a) Raman spectra of as-fabricated and $\mathrm{NH}_{3}$ plasmatreated graphene. The insert shows an optical microscopy image of the as-fabricated graphene. (b) and (c) AFM images show surface morphology of the as-fabricated and plasmatreated graphene. Line profiles are inserted as dashed lines.

\section{[10].}

To investigate the effect of annealing temperature systematically, several graphene samples were plasmadoped and successively vacuum-annealed at different temperatures. Representative Raman spectra recorded from the same location are shown in Fig. 2. As shown in Fig. 1(a), the existence of D- and D'-bands is confirmed as well as the increase in the $\mathrm{I}_{\mathrm{D}} / \mathrm{I}_{\mathrm{G}}$ and $\mathrm{I}_{\mathrm{G}} / \mathrm{I}_{2 \mathrm{D}}$ values after plasma treatment. A three-stage model can be employed to predict the evolution of the graphite structures [14,15]. According to the model, the intensity ratio of $\mathrm{I}_{\mathrm{D}} / \mathrm{I}_{\mathrm{G}}$ in the Raman spectra increases when the graphite structure transforms into a nanocrystal graphite structure during stage 1 (as is the case in this study). In addition, the crystallite size $\left(\mathrm{L}_{\mathrm{a}}\right)$ of the structures can be estimated using Eq. $1[15,16]$.

$$
\mathrm{L}_{\mathrm{a}}=\left(2.4 \times 10^{-10}\right) \times \lambda^{4} \times\left(\mathrm{I}_{\mathrm{G}} / \mathrm{I}_{\mathrm{D}}\right)
$$

where, $\lambda$ is the excitation wavelength of the Raman laser. The variations of the $\mathrm{I}_{\mathrm{D}} / \mathrm{I}_{\mathrm{G}}$ and $\mathrm{I}_{\mathrm{G}} / \mathrm{I}_{2 \mathrm{D}}$ values estimated from Raman spectra in Fig. 2 are summarized in Figs. 3(a) and $3(\mathrm{~b})$. The $\mathrm{I}_{\mathrm{D}} / \mathrm{I}_{\mathrm{G}}$ and $\mathrm{I}_{\mathrm{G}} / \mathrm{I}_{2 \mathrm{D}}$ values increase after the plasma treatment due to the structural defects induced by plasma ion bombardment. Then, the $\mathrm{I}_{\mathrm{D}} / \mathrm{I}_{\mathrm{G}}$ and $\mathrm{I}_{\mathrm{G}} / \mathrm{I}_{2 \mathrm{D}}$ values decrease after vacuum annealing. It is interesting that the reduction in the $\mathrm{I}_{\mathrm{D}} / \mathrm{I}_{\mathrm{G}}$ and $\mathrm{I}_{\mathrm{G}} / \mathrm{I}_{2 \mathrm{D}}$ values becomes greater as the annealing temperature increases. For instance, the $\mathrm{I}_{\mathrm{D}} / \mathrm{I}_{\mathrm{G}}$ and $\mathrm{I}_{\mathrm{G}} / \mathrm{I}_{2 \mathrm{D}}$ values for graphene annealed at $100{ }^{\circ} \mathrm{C}$ are 0.47 and 0.63 , respectively, while the same values for graphene annealed at $500{ }^{\circ} \mathrm{C}$ decrease to 0.12 and 0.38 , respectively. According to Eq. 1, $\mathrm{L}_{\mathrm{a}}$ of the plasma-treated sample is 


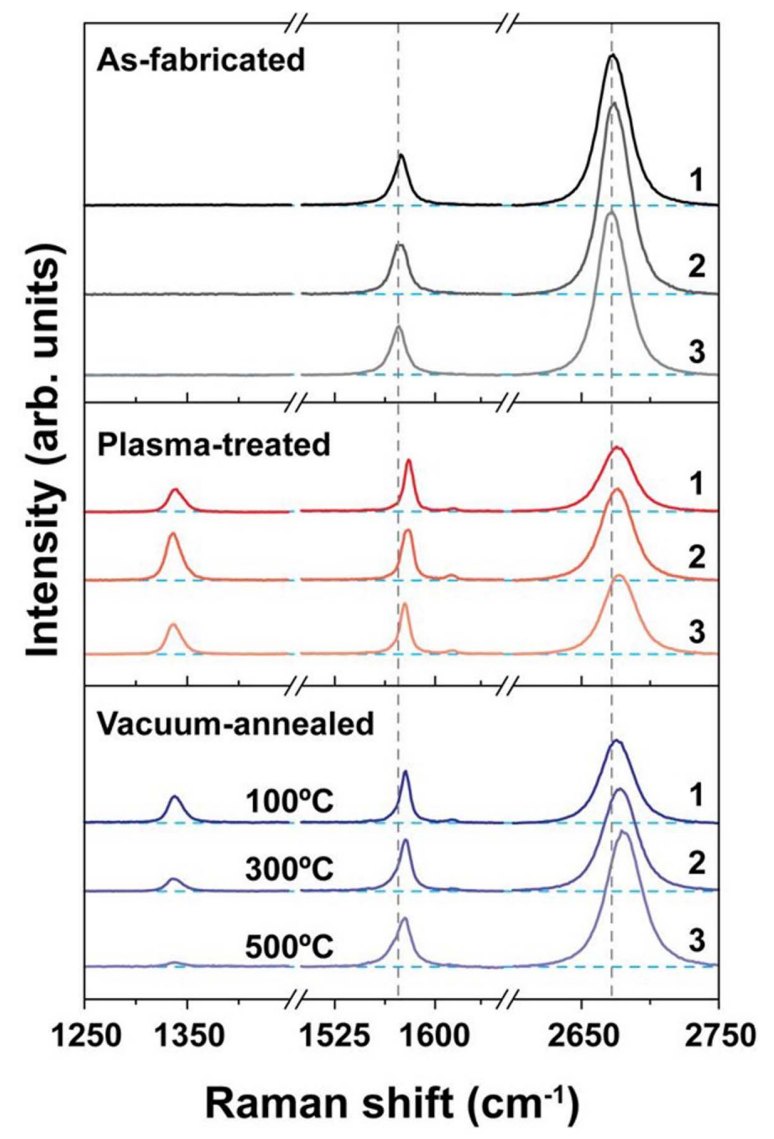

Figure 2. Raman spectra of as-fabricated, $\mathrm{NH}_{3}$ plasma-treated and vacuum-annealed graphene of three samples, respectively. The $\mathrm{NH}_{3}$ plasma treatment was performed under the same environment of 0.3 Torr, $450 \mathrm{~V}, 0.45 \mathrm{~W}$ and $10 \mathrm{~s}$. Samples 1,2 and 3 were annealed for $1 \mathrm{~h}$ under $10^{-6}$ Torr at $100{ }^{\circ} \mathrm{C}, 300{ }^{\circ} \mathrm{C}$ and $500{ }^{\circ} \mathrm{C}$, respectively.

$13.87 \mathrm{~nm}$ and increases to 14.73 and $29.15 \mathrm{~nm}$ after vacuum annealing at 100 and $500{ }^{\circ} \mathrm{C}$, respectively. This implies that the plasma-treated graphene might be healed structurally by high vacuum annealing and the higher annealing temperature would be suitable for structural reorganization of damaged graphene.

In addition to the structural damage and recovery, it is known that a change in G-band $\left(\Delta \omega_{G}\right)$ and full width at half maximum of G-band $\left(\mathrm{FWHM}_{\mathrm{G}}\right)$ are closely related to charge doping and structural change, respectively. These factors from the Raman spectra, $\Delta \omega_{G}$ and $\mathrm{FWHM}_{\mathrm{G}}$ have been adopted to estimate the level of charge-doping and annealing-induced structural recovery. Yan et al. also reported the upshift of $\omega_{G}$ and an increase in the $\mathrm{FWHM}_{\mathrm{G}}$ in electrically-doped graphene [17]. The doping level can be calculated using the relationship between the $\Delta \omega_{G}$ and the Fermi level $\left(\mathrm{E}_{\mathrm{F}}\right)$ as follows:

$$
\hbar \omega_{\mathrm{G}}-\hbar \omega_{\mathrm{G}}^{0}=\lambda\left\{\left|\mathrm{E}_{\mathrm{F}}\right|+\frac{\hbar \omega_{\mathrm{G}}}{4} \ln \left|\frac{2\left|\mathrm{E}_{\mathrm{F}}\right|-\hbar \omega_{\mathrm{G}}}{2\left|\mathrm{E}_{\mathrm{F}}\right|-\hbar \omega_{\mathrm{G}}}\right|\right\}
$$

Figure 4(a) displays the Raman spectra showing a magnified G-band of the as-fabricated, plasma-treated and vacuum-annealed graphene. The red-shift of the G-band
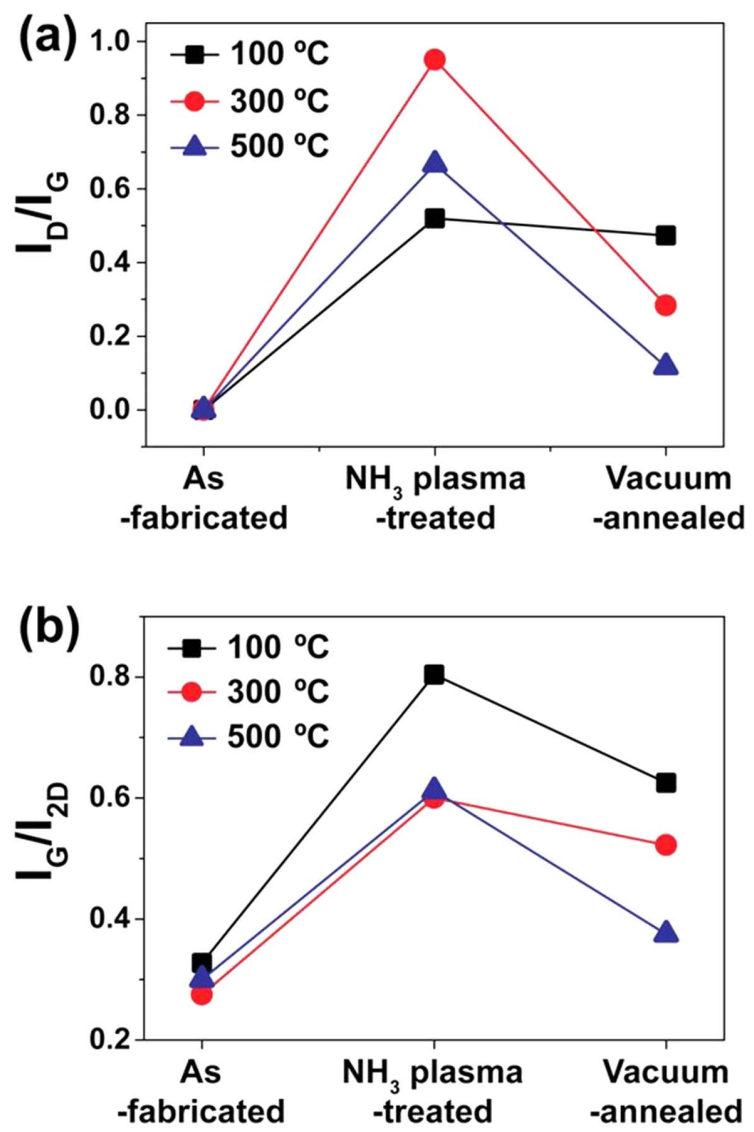

Figure 3. (a) and (b) graphs show the variation in $\mathbf{I}_{\mathbf{D}} / \mathbf{I}_{\mathbf{G}}$ and $\mathbf{I}_{G} /$ $I_{2 D}$ measured from Fig. 2.
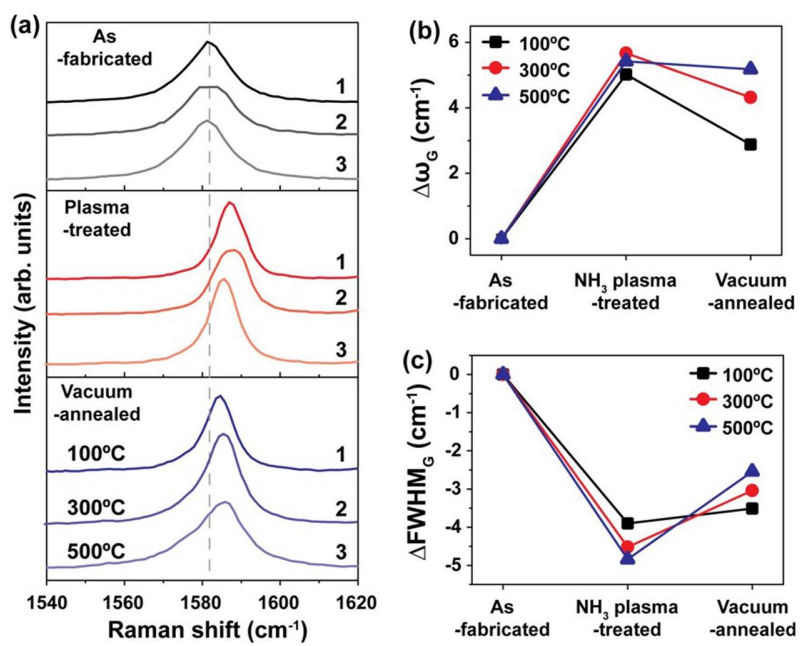

Figure 4. (a) Detailed view of the Raman spectra near the Gband of as-fabricated, $\mathrm{NH}_{3}$ plasma-treated and vacuumannealed graphene. (b) and (c) graphs show $\Delta \omega_{G}$ and $\Delta \mathrm{FWHM}_{\mathrm{G}}$ measured from (a), respectively.

and the decrease in the $\mathrm{FWHM}_{\mathrm{G}}$ are observed after plasma treatment. Their quantitative variation is summarized in Figs. 4(b) and 4(c). Specifically, the $\omega_{\mathrm{G}}$ and $\mathrm{FWHM}_{\mathrm{G}}$ of the as-fabricated graphene are 1582 and $15.1 \mathrm{~cm}^{-1}$, respectively, with an average shift of $5.36 \mathrm{~cm}^{-1}\left(\Delta \omega_{\mathrm{G}}\right)$ and $4.40 \mathrm{~cm}^{-1}$ $\left(\Delta \mathrm{FWHM}_{\mathrm{G}}\right)$ after plasma treatment. Based on Eq. 2 , the 


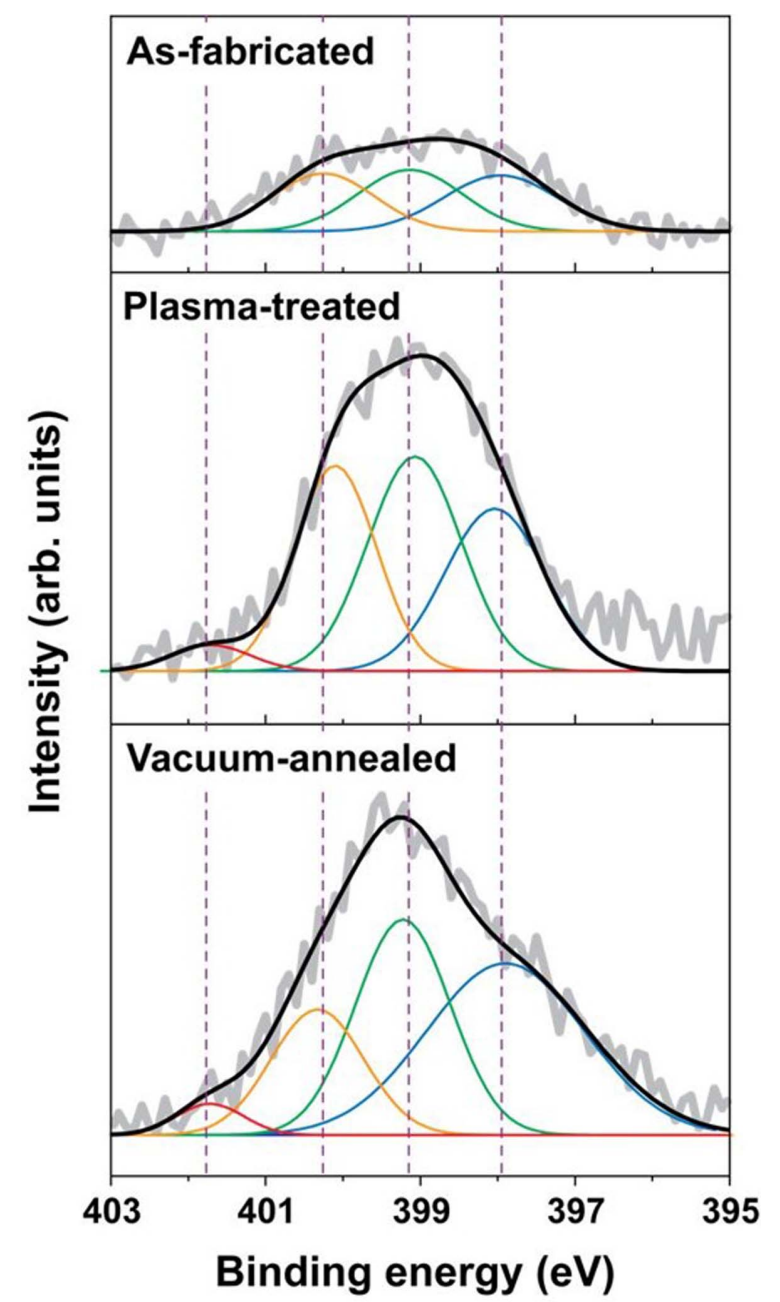

Figure 5. N1s peak of XPS spectrum and its fitted sub-peaks of as-fabricated, plasma-treated and vacuum-annealed graphene.

doping level of the plasma-treated graphene under optimum conditions can be estimated to be $2.2 \times 10^{12} \mathrm{~cm}^{-2}$. This value is very similar to other doping results reported indicates the method used here is effective for graphene doping $[18,19]$.

After vacuum annealing, it should be noted that the $\omega_{\mathrm{G}}$ and $\mathrm{FWHM}_{\mathrm{G}}$ values are similar to the initial values of pristine graphene, implying de-doping of the doped species. However, based on the large difference in $\omega_{\mathrm{G}}$ and $\mathrm{FWHM}_{\mathrm{G}}$ values between the pristine and annealed samples, it can be assumed that most of the doping effect remains after vacuum annealing. Interestingly, the $\Delta \omega_{\mathrm{G}}$ increases and $\Delta \mathrm{FWHM}_{\mathrm{G}}$ decreases with increasing annealing temperature. For instance, $\Delta \omega_{\mathrm{G}}$ gradually increases to $3.12,4.32$ and $4.78 \mathrm{~cm}^{-1}$ after 100,300 and $500{ }^{\circ} \mathrm{C}$ annealing, respectively. In addition, $\Delta \mathrm{FWHM}_{\mathrm{G}}$ continually decreases to $3.71,3.27$ and $2.57 \mathrm{~cm}^{-1}$ after 100 , 300 and $500{ }^{\circ} \mathrm{C}$ annealing, respectively. To sum up, it can be concluded that plasma-doped graphene can be structurally recovered, while maintain its doping effect, even after high temperature annealing at $500^{\circ} \mathrm{C}$.
To investigate the bonding configuration of the nitrogen atoms to the carbon network, an XPS analysis was performed using a graphene sample prepared via chemical vapor deposition, owing to the small size of exfoliated graphene [20]. There are subpeaks corresponding to graphitic $\mathrm{N}(401.7 \mathrm{eV})$, pyrrolic $\mathrm{N}(400.1 \mathrm{eV})$, amino groups $(399.1 \mathrm{eV})$, and pyridinic $\mathrm{N}(398.5 \mathrm{eV})$, corresponding to nitrogen bonding to carbon networks. The N1s XPS peaks from the as-fabricated, plasma-treated, and vacuumannealed graphene are presented in Fig. 5. For the plasmadoped graphene, the peaks for pyrrolic, pyridinic, and amino bonds are pronounced. Generally, it has been known that pyrrolic $\mathrm{N}$ is formed more easily damaged graphene than pyridinic and graphitic $\mathrm{N}$ because there are many dangling bonds of carbon atoms in the damaged graphene structure. Interestingly, the peak area for pyrrolic $\mathrm{N}$ is greatly reduced in contrast to the stable pyridinic component after vacuum annealing at $500^{\circ} \mathrm{C}$. This is direct evidence of the healing of damaged graphene structures due to plasma treatment. Furthermore, based on the theoretical results of Schiros et al. [21], the annealed graphene would be still electron-doped, as the pyridinic component acts as an electron donor. The doping level can also be estimated from the XPS spectra, through the intensity ratio of the $\mathrm{N} 1 s$ peak to the $\mathrm{C} 1 s$ peak. The intensity ratio of $\mathrm{N} 1 s / \mathrm{C} 1 s$ after plasma treatment increases to $6 \%$ and still maintains $5 \%$ after the annealing, even at $500{ }^{\circ} \mathrm{C}$, indicating the stability of the doping components.

\section{Conclusions}

The behavior of plasma-doped graphene on high temperature vacuum annealing has been demonstrated in this study. Graphene samples were vacuum annealed with ammonia plasma-treatment at elevated temperatures. It was found that the crystallite size of the plasma-treated samples increases after vacuum annealing and the degree of the structural healing is enhanced with increased annealing temperatures. The nitrogen doping level after annealing is similar to the plasma-doped sample, indicating the high doping stability. After vacuum annealing, the presence of a pyrrolic binding component greatly decreases with respect to the other configurations. Finally, it can be concluded that the plasma-doped graphene can be structurally restored by high vacuum annealing at elevated temperatures, without the loss of the functional doping effect.

\section{Acknowledgements}

This research was supported by Basic Science Research Program through the National Research Foundation of Korea (NRF) funded by the Ministry of Education (Grant No. 2017081575). 


\section{References}

[1] K. S. Novoselov, A. K. Geim, S. V. Morozov, D. Jiang, M.I. Katsnelson, I. V. Grigorieva, S. V. Dubonos, and A. A. Firsov, Nature 438, 197 (2005).

[2] J. H. Chen, C. Jang, S. Xiao, M. Ishigami, and M. S. Fuhrer, Nat. Nanotechnol. 3, 206 (2008).

[3] E. Silva, A. R. B. Mendez, Z. M. Barnett, X. Jia, M. S. Dresselhaus, H. Terrones, M. Terrones, B. G. Sumpter, and V. Meunier, Phys. Rev. Lett 105, 045501 (2010).

[4] X. Li, X. Wang, L. Zhang, S. Lee, and H. Dai, Science 319, 1229 (2008).

[5] X. Yang, S. Tang, G. Ding, X. Xie, M. Jiang, and F. Huang, Nanotechnol. 23, 025704 (2012).

[6] L. Jiao, L. Zhang, X. Wang, G. Diankov, and H. Dai, Nature 458 (2009) 877-880.

[7] X. Li, H. Wang, J. T. Robinson, H. Sanchez, G. Diankov, and H. Dai, J. Am. Chem. Soc. 13115939 (2009).

[8] Y. C. Lin, C. Y. Lin, and P. W. Chiu, Appl. Phys. Lett. 96, 133110 (2010).

[9] L. Xie, L. Jiao, and H. Dai, J. Am. Chem. Soc. 132, 14751 (2010).

[10] B. J Lee, D. H. Shin, S. Lee, and G. H. Jeong, Carbon 123, 174 (2017).
[11] S. I. Jo and G. H. Jeong, Appl. Sci. Converg. Technol. 24, 262 (2015).

[12] A. C. Ferrari, J. C. Meyer, V. Scardaci, C. Casiraghi, M. Lazzeri, F. Mauri, S. Piscanec, D. Jiang, K. S. Novoselov, S. Roth, and A.K. Geim, Phys. Rev. Lett. 97, 187401 (2006).

[13] Z. Ni, Y. Wang, T. Yu, and Z. Shen, Nano Res. 1, 273 (2008).

[14] A.C. Ferrari and J. Robertson, Phys. Rev. B 61, 14059 (2000).

[15] A.C. Ferrari, Solid State Commun. 143, 47 (2007).

[16] O. A. Maslova, M. R. Ammar, G. Guimbretiere, J. N. Rouzaud, and P. Simon, Phy. Rev. B 85, 134205 (2012).

[17] J. Yan, Y. Zhang, P. Kim, and A. Pinczuk, Phys. Rev. Lett. 98, 166802 (2007).

[18] C. Stampfer, F. Molitor, D. Graf, K. Ensslin, A. Jungen, C. Hierold, and K. Ensslin, Appl. Phys. Lett. 91, 241907 (2007).

[19] Y. P. Lin, Y. Ksari, D. Aubel, S. H. Garreau, G. Borvon, Y. Spiegel, L. Roux, L. Simon, and J. M. Thermlin, Carbon 100, 337 (2016).

[20] B. J. Lee and G. H. Joeng, Appl. Phys. A 116, 15 (2014).

[21] T. Schiros, D. Nordlund, L. Pálová, D. Prezzi, L. Zhao, K. S. Kim, U. Wrstbauer, C. Gutierrez, D. Delongchamp, C. Jaye, D. Fisher, H. Ogasawara, L. G. M. Pettersson, D. R. Reichman, P. Kim, M. S. Hybertsen, and A. N. Pasupathy, Nano Lett. 12, 4025 (2012). 